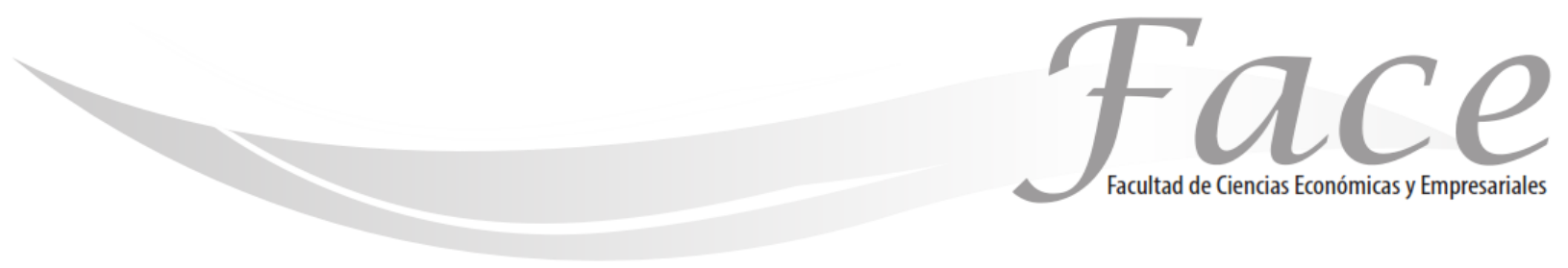

ISSN Impreso: 1794-9920

ISSN Electrónico: 2500-9338

Volumen $16-\mathrm{N}^{\circ} 2$

Año 2016

Págs. 90 - 101

\title{
RELACIÓN ENTRE DESEMPEÑO FINANCIERO Y RESPONSABILIDAD SOCIAL EMPRESARIAL: UN ANÁLISIS DE LITERATURA (1984-2013)*
}

\author{
Carlos Hernán Cubillos Calderón ** \\ Enlace ORCID: http://orcid.org/0000-0001-5171-6285 \\ Omar Giovanni Rosero Villabón *** \\ Enlace ORCID: http://orcid.org/0000-0003-3193-5338
}

Fecha de Recepción: 7 de Junio 2016

Fecha de Aprobación: 12 de Octubre 2016

\section{Resumen:}

Investigar la relación entre responsabilidad social empresarial (RSE) y desempeño financiero (DF) es una labor que lleva alrededor de tres décadas, producto de la cual se cuentan 186 trabajos relacionados en más de 25 meta análisis o estudios de resúmenes, siendo el presente artículo el primero en español. Se seleccionaron 30 investigaciones científicas publicadas en revistas de orden internacional, de las que se mencionan propósito, muestra poblacional y resultados. Un importante número de estas investigaciones coincide en una relación positiva entre la RSE y el DF, algunas consideran que la relación no es relevante y solo una señala que es negativa.

Palabras Claves: Responsabilidad social empresarial, desempeño financiero, investigaciones

\footnotetext{
* Artículo resultado del proyecto de investigación titulado "Relación entre el desempeño financiero y responsabilidad social empresarial: un análisis en la banca europea", financiado por la Universidad del Tolima bajo el código 20312; que a su vez corresponde a la tesis doctoral presentada a la Universidad de Valladolid, la cual fue dirigida por el Dr. José Miguel Rodríguez Fernández.

** Administrador Financiero de la Universidad del Tolima, Magíster en Administración de la Universidad Nacional de Colombia, Profesor de Planta de la Facultad de Ciencias Económicas y Administrativas e Investigador del Grupo de Investigación en Pensamiento Estratégico e Innovación Empresarial -GIPE- de la Universidad del Tolima. Contacto: chcubi@ut.edu.co

** Administrador de Empresas de la Universidad del Tolima, Magíster en Administración de la Universidad Nacional de Colombia, Doctor en Gestión y Administración de Empresas de la Universidad de Valladolid, Coordinador del Grupo de Investigación en Finanzas -GRUPINFINUT- de la Universidad del Tolima. Contacto: orosero@ut.edu.co
} 


\title{
RELATIONSHIP BETWEEN CORPORATE SOCIAL RESPONSIBILITY AND FINANCIAL PERFORMANCE: A LITERATURE REVIEW (1984-2013)
}

\begin{abstract}
:
Over the past three decades research on the relationship between corporate social responsibility (CSR) and financial performance (FP) has produced 186 related works in more than 25 meta-analysis or study summaries, being this the first article in Spanish. A total of 30 scientific research papers published in international journals, that emphasized on purpose, population sample and results were selected. A significant number of these studies coincide that there is a positive relation between CSR and FP; some consider that this relationship is not relevant and only one indicates that it is negative.
\end{abstract}

Keywords: Corporate social responsibility, financial performance, research

\section{RELAÇÃO ENTRE RESPONSABILIDADE SOCIAL EMPRESARIAL E DESEMPENHO FINANCEIRO: UMA ANÁLISE DA LITERATURA (1984-2013)}

\begin{abstract}
Resumo:
A relação entre a responsabilidade social empresarial (RSE) e o desempenho financeiro (DF) tem sido objeto de investigação nas ultimas três décadas, com 186 trabalhos relacionados em mais de 25 meta-análise e estudo de resumos, sendo este artigo 0 primeiro em espanhol. Foram selecionados 30 trabalhos de pesquisa científica publicados em revistas internacionais, que enfatizava o propósito, amostra populacional e os resultados. Um número significativo desses estudos coincide que existe uma relação positiva entre a RSE e DF; alguns consideran que essa relação não é relevante e apenas um indica que é negativo.
\end{abstract}

Palavras-Chave: Responsabilidade social empresarial, desempenho financeiro, pesquisa. 


\section{INTRODUCCIÓN:}

Algunos autores consideran que el origen de la responsabilidad social empresarial está estrechamente ligado con el origen mismo de los negocios. Dicha afirmación surge del conocimiento de leyes que se remontan a unos 5.000 años, promulgadas con el propósito de proteger los bosques o al código del rey Hammurabi, introducido en la antigua Mesopotamia en 1.700 a. c., en el cual los constructores, posaderos o agricultores serían condenados a muerte si su negligencia había ocasionado grandes molestias a los ciudadanos locales. De manera similar, en la Roma antigua los senadores se quejaban de la falta de empresas que pagaran impuestos suficientes para financiar campañas militares.

Sin embargo, se puede considerar que la responsabilidad social empresarial, como concepto moderno, se empezó a desarrollar a partir de la segunda década del siglo XX, con el surgimiento de la gran empresa corporativa. Durante esta década, los Estados Unidos de Norteamérica enfrentaron un crecimiento económico sin precedentes el cual trajo consigo cambios sociales como la urbanización, el aumento de la riqueza material y el desarrollo de una cultura empresarial. En consecuencia, se promovió la relación entre las características institucionales de las grandes empresas y del "nacimiento" de ciertas responsabilidades 0 acciones de estas empresas para con la sociedad

En las últimas décadas la responsabilidad social empresarial, como campo de estudio, ha cobrado tal relevancia que académicos de diversas latitudes vienen hablando, investigando y escribiendo sobre responsabilidad social empresarial o responsabilidad social corporativa, que en términos sencillos se entiende como la forma en que una organización empresarial se relaciona con la sociedad con el propósito de generar beneficios para ambas partes. Esto implica la inclusión de las expectativas de todos los grupos de interés a fin de generar una relación gana - gana entre la organización y sus stakeholders, participantes o implicados.

Los investigadores han propuesto vínculos teóricos entre lo económico, lo social y lo ecológico, en el entendido que "una empresa que crea valor no solo beneficia a sus accionistas, sino también el valor de todos los derechos de las otras partes involucradas, mientras que, en cambio, todas las partes involucradas se resienten cuando la dirección fracasa en la creación de valor para el accionista" (Rappaport, 2006, p. 28).

De manera particular, se ha intentado comprobar la existencia de una relación positiva entre la responsabilidad social empresarial y el desempeño financiero sin lograr resultados totalmente concluyentes. Es probable que la razón última de que esto ocurra guarde relación con una cuestión de fondo: el problema de medir apropiadamente la responsabilidad social de una organización, un problema hasta ahora no bien resuelto.

Así, las investigaciones adelantadas no han coincidido totalmente en sus resultados, toda vez que un número importante revela alta presencia de una relación positiva entre la responsabilidad social empresarial y el desempeño financiero, unos pocos manifiestan que esa relación no es fuerte o relevante; y tan solo en uno de ellos se señala que es negativa. Esta falta de uniformidad en los resultados, probablemente, genera que las organizaciones no realicen inversiones en prácticas de RSE que contribuyan al logro de objetivos estratégicos.

\section{MARCO TEÓRICO:}

Uno de los primeros enfoques académicos de la responsabilidad social empresarial -RSE- fue desarrollado tras la Segunda Guerra Mundial por Howard Bowen, quien señaló que la prosperidad de las empresas americanas de la postguerra debería llevar ciertas obligaciones sociales. Este estudioso de la ética y la conducta empresarial elaboró una de las primeras teorizaciones de la RSE tal y como hoy se conoce.

Para el trabajo investigativo que dio origen al presente artículo se consideró relevante el planteamiento de Carroll (1991) a través del cual se establecen cuatro tipos de responsabilidades que los empresarios deben poseer: las responsabilidades económicas que consisten en que la empresa debe lograr utilidades financieras, es decir, debe generar lucro para sus dueños y/o accionistas.

Las responsabilidades legales se centran en el cumplimiento de todos los requerimientos legales que existan a nivel internacional, nacional y regional, es decir, la empresa debe realizar sus misiones económicas en el marco de la Ley. Las responsabilidades éticas, a su vez, se refieren a los comportamientos que debe tener la empresa que si bien no están normadas son aquellas que son esperadas o prohibidas por la comunidad. Por último, las responsabilidades filantrópicas abarcan aquellas acciones corporativas que responden a las expectativas que la sociedad tiene de las empresas en su rol de buenos ciudadanos corporativos. 
Por su parte, Cadbury (2006) establece que existe un nivel primario en el que la empresa se compromete a cumplir sus responsabilidades básicas de retribuir a sus empleados, pagar a sus proveedores y sus préstamos, remunerar a sus accionistas, etc. El incumplimiento de estas responsabilidades es relativamente fácil de determinar debido a que da lugar a las sanciones previstas en las leyes. En el nivel secundario, las empresas deben preocuparse por los impactos que produce su actividad en su entorno y evitar el daño medioambiental. En este nivel no sólo se debe cumplir con los mínimos establecidos, sino algo más. Finalmente, en el nivel terciario es aquel en el que la empresa debe preguntarse acerca de cómo puede influir positivamente en la sociedad.

Por otra parte, el objetivo básico financiero de las empresas, y por consiguiente de los directivos y sus colaboradores consiste en maximizar el patrimonio de los inversionistas. La riqueza de los propietarios se mide por el precio de las acciones, que a su vez se basa en la oportunidad de los rendimientos (flujos de efectivo), su magnitud y su riesgo. "Al considerar cada alternativa de decisión 0 acción financiera posible en términos de su impacto sobre el precio de las acciones de la empresa, los administradores financieros solo deben aceptar aquellas actividades que se espera incrementen el precio de las acciones" (Gitman y Zutter, 2012, p. 9).

La gerencia financiera, por tanto, está llamada a dinamizar los mecanismos que permitan generar, analizar y evaluar el desempeño financiero logrado mediante la asignación de recursos en actividades internas y de relación con el entorno empresarial. Bajo esta perspectiva, el concepto de valor presenta gran complejidad al tratar de entenderlo desde la vida práctica, pero se puede decir que se compendia en el valor de mercado de cada acción 0 aporte social, y que, "de alguna manera, a través del tiempo, el valor promedio de la acción refleja el éxito o fracaso de la gestión financiera" (Ortiz, 2015, p. 21).

Aunque teóricamente se plantean relaciones positivas entre la responsabilidad social empresarial y el desempeño financiero y un buen número de organizaciones destinan recursos a prácticas de RSE, directivos de otras organizaciones no están a favor de implementarlas toda vez que consideran que "destinar recursos y esfuerzos a iniciativas que no forman parte de la actividad principal de la organización podría comprometer su buena marcha financiera" Jensen (2001) y Sundaram \& Inkpen (2004) citados en Aguilera, Guerrero y Morales (2015, p. 420), posturas que en gran medida motivaron el desarrollo de la investigación que dio origen a este documento.

\section{METODOLOGÍA:}

Las investigaciones empíricas que se han realizado con el propósito de determinar las relaciones entre el desempeño social y el desempeño financiero han nacido a raíz de los siguientes cuestionamientos: ¿La realización de acciones de RSE inciden en el desempeño financiero de las empresas? y iel desempeño financiero de una organización define el nivel de acciones de RSE que realiza una organización? Para dar respuesta a estas inquietudes se propuso una hipótesis de investigación que considera una relación positiva entre las variables analizadas.

Esta hipótesis presentada en primer lugar por Preston y O'Bannon (1997), y utilizada en variados estudios empíricos como, Gómez (2008), Makni, Francoeur y Bellavance (2009), García, Campillo y Sánchez (2010), Setó y Angla (2011), entre otros, sugiere que niveles más altos de RSE conducen directamente a niveles más altos de desempeño financiero (DF), dado que los costos que suponen desarrollar dichas iniciativas resultan más que compensados por los beneficios que se pueden obtener.

Hipótesis H1: DF $=\mathrm{f}(\mathrm{RSE})$ donde $\mathrm{dDF} / \mathrm{dRSE}>0$. Altos (bajos) niveles de acciones de responsabilidad social empresarial, conducen a unos altos (bajos) niveles de desempeño financiero.

A partir de la definición de la hipótesis se realizó un rastreo de estudios publicados en revistas internacionales, que consideraran relaciones entre responsabilidad social empresarial y desempeño financiero en términos de propósito, muestra poblacional y resultados. Producto de esta revisión se seleccionaron 30 investigaciones que aparecen relacionadas en el desarrollo teórico y que hacen parte del marco teórico de la investigación denominada Relación entre desempeño financiero y responsabilidad social empresarial: un análisis en la banca europea realizada entre los años 2011 y 2015.

\section{DESARROLLO TEÓRICO}

Entre los años 1970 y 1979, Cochran y Wood (1984) realizaron un estudio con 75 empresas estadounidenses obtenidas de los registros de la agencia de calificación de riesgo Standard \& Poors, mediante el cual se determinó que la variable financiera más fuertemente correlacionada con la responsabilidad social empresarial es la edad de los activos, y que la omisión de esta variable en estudios anteriores pudo haber generado falsas correlaciones 
positivas entre la responsabilidad social empresarial y los resultados financieros. Sin embargo, aún con esta variable incluida no se generó evidencia contundente de una correlación positiva entre la responsabilidad social empresarial y el desempeño financiero.

A su vez, Aupperle, Carroll y Hatfield (1985) adelantaron una investigación, con 241 empresas del directorio de Forbes en 1981, con el propósito de desarrollar un instrumento para medir el grado de orientación corporativa hacia la responsabilidad social, a partir de un modelo que define la responsabilidad social empresarial propuesto por Carroll (1979), en el que se articulan componentes económicos, legales, éticos y filantrópicos. Encontraron que, aparentemente, existe una fuerte relación inversa entre los componentes económicos y éticos. Adicionalmente, las fuertes correlaciones negativas entre lo económico y cada uno de los tres componentes no económicos sugieren que una empresa se orienta sobre todo hacia los aspectos económicos; y con menor énfasis hacia los aspectos éticos, legales y filantrópicos. Un hallazgo particularmente interesante, dado el hecho de que los tres componentes no económicos presentaron correlaciones modestas 0 insignificantes entre ellos mismos; así, las correlaciones entre estos componentes mostraron pocas relaciones sustantivas.

McGuire, Sundgren y Schneeweis (1988), a su vez, trabajaron con 131 empresas estadounidenses de diferentes sectores que se encontraban en la encuesta anual de reputación corporativa de la revista Fortune, entre los años 1983 y 1985. El estudio sugirió que las empresas débiles en materia de responsabilidad social también experimentan más baja rentabilidad sobre los activos y rendimiento bursátil total que aquellas empresas fuertes en responsabilidad social. A su vez, las medidas de rentabilidad basadas en la contabilidad, particularmente la rentabilidad sobre los activos, demostraron ser mejores predictores de la responsabilidad social empresarial que las medidas de rentabilidad basadas en el mercado bursátil. Los resultados mostraron, además, que la reducción de riesgo en la empresa es un importante beneficio de la responsabilidad social.

Entre los años 1989 a 1991, Waddock y Graves (1997) realizaron un estudio a partir de una muestra de 469 empresas estadounidenses de 13 sectores, cuyos resultados mostraron que el desempeño social empresarial depende del previo desempeño financiero, con un signo de la relación positivo y significativo. $Y$ caso contrario: en la mayor parte de los modelos estimados; el desempeño financiero depende de forma positiva y significativa del índice previo de desempeño social. Así, parece que la disponibilidad de recursos facilita gastar estos recursos en "hacer el bien haciéndolo bien", dando lugar a una mejora en el desempeño social empresarial (teoría del excedente de recursos). A su vez, esta mejora conduce a mayor desempeño financiero (teoría de la buena gestión).

De otro lado, Preston y O'Bannon (1997) analizaron 67 grandes empresas de Estados Unidos con los datos obtenidos de una combinación entre la revista Fortune y COMPUSTAT, entre los años 1982 y 1992, y observaron fuertes correlaciones positivas entre ambos tipos de desempeño en las combinaciones de periodos vigentes y de periodos anteriores. De las 270 correlaciones computadas, no hay una sola relación negativa y significativa entre los tres indicadores de desempeño social (compromiso con la comunidad y responsabilidad ambiental, capacidad para seleccionar y retener buen personal, calidad de productos y servicios) y los tres indicadores de desempeño financiero (rentabilidad sobre activos, rentabilidad sobre patrimonio neto, rentabilidad sobre la inversión).

Ruf y colaboradores (2001) tras estudiar 488 empresas estadounidenses de diversos sectores seleccionadas de la base de datos de KLD, con la información financiera de COMPUSTAT para el periodo 1991 a 1995, generaron resultados que proporcionan cierto apoyo a uno de los principios de la teoría stakeholders que afirma que el grupo de interés dominante, los accionistas, financieramente se benefician cuando la gestión satisface las demandas de múltiples grupos de interés. Específicamente, el cambio en la calificación del desempeño social empresarial aparece positivamente asociado con el crecimiento en las ventas para el año actual y el siguiente.

Ziegler, Rennings y Schröder (2002) investigaron 214 empresas europeas de diversos sectores, con información de la base de datos de Thomson Financial Datastream para el periodo de 1996 a 2001. El resultado más importante del análisis econométrico correspondió al significativo efecto positivo de un desempeño medioambiental sectorial creciente sobre el promedio del retorno mensual de las acciones de las empresas estudiadas. La influencia más fuerte puede ser identificada por las dos más altas evaluaciones del desempeño medioambiental sectorial comparadas con las evaluaciones más bajas. En contraste, un incremento en el desempeño social del sector tiene un efecto negativo sobre el valor de las acciones.

Los investigadores D'Arcimoles y Trébucq (2003) presentaron los primeros resultados obtenidos sobre la relación entre responsabilidad social empresarial y desempeño económico en 99 empresas francesas pertenecientes al índice SBF 120 de la Bolsa de Valores Francesa. El estudio permitió concluir que en las empresas francesas existiera una ausencia total de relaciones significativas entre el desempeño financiero y desempeño 
social, sino la posibilidad que las empresas que muestran un mejor desempeño social puedan obtener algunos beneficios económicos. Por otra parte, la hipótesis del oportunismo de los directivos no pudo ser totalmente rechazada, ya que es probable que algunos de los responsables de las empresas, una vez alcanzado un alto nivel de rendimiento financiero, se pueden beneficiar al reducir sus compromisos de colaboración frente a clientes, proveedores 0 accionistas.

A su vez, Barnett y Salomon (2006) se propusieron avanzar en el debate sobre la relación entre el desempeño financiero y el desempeño social dentro de los fondos de inversión que practican la inversión socialmente responsable, para ello trabajaron con 61 fondos estadounidenses de inversión, cuya información fue recolectada a partir del Foro de Inversión Social que proporcionó la información del número y tipo de acciones sociales, del Center of Research in Security Prices y servicios de análisis y seguimiento de fondos de inversión. Con el estudio que cubrió el periodo 1972 a 2000 se logró determinar que a medida que aumenta el número de filtros sociales utilizados por los fondos de inversión socialmente responsables para elegir sus colocaciones de recursos, los retornos financieros tienen forma de $U$ : en un primer momento se reducen, pero después se incrementan hasta que ese número de filtros alcanza el máximo. Se encontró, además, que el desempeño financiero varía de acuerdo con los tipos de filtros sociales aplicados, y que no se puede plantear una relación significativa entre los filtros en las relaciones laborales y el rendimiento financiero ajustado al riesgo. Sin embargo, los fondos de inversión que seleccionaron empresas por su cumplimiento de las normas de igualdad en el empleo sufrieron una reducción en su desempeño financiero, en comparación con el fondo de referencia. Las mejores relaciones con la comunidad parecen conducir a una mejor performance del fondo. Finalmente, en contra de lo esperado, los filtros sobre la base de criterios medioambientales se encontraron negativamente relacionados con el rendimiento ajustado al riesgo.

Bouquet y Deutsch (2008) se propusieron investigar, en 813 empresas multinacionales de 1991 a 2003 a partir de la información sobre responsabilidad social proveniente de la base de datos KLD y la información financiera de Standard \& Poor's Research Insight, la relación entre el desempeño social empresarial y la multinacionalidad de la empresa, definida como la capacidad de capturar ventas rentables en mercados extranjeros; en segundo lugar, examinar si el desempeño social empresarial modera la relación entre I + D y multinacionalidad, bien establecida en la literatura sobre negocios internacionales. Así, se determinó que el desempeño social empresarial modera la relación entre investigación y desarrollo y multinacionalidad; cuando el desempeño social de una empresa es considerado altamente positivo, la relación positiva entre investigación y desarrollo y multinacionalidad es más fuerte. No obstante, cuando el desempeño social de una empresa es calificado como altamente negativo, la relación positiva entre investigación y desarrollo y la multinacionalidad también es más fuerte. Aunque hay costos importantes asociados con la adopción y la implementación internacional de estándares de desempeño social empresarial, los altos niveles de desempeño social aún tienen un efecto positivo sobre la multinacionalidad de una empresa. Sin embargo, los niveles intermedios de desempeño social no respaldan los esfuerzos de multinacionalidad de la empresa, incluso en comparación con empresas que tienden a ignorar sus responsabilidades sociales.

Es importante mencionar, además que Saleh, Zulkiflib y Muhamad (2008) estudiaron las 200 empresas más grandes cotizantes en la Bolsa de Valores de Malasia durante el periodo de 2000 a 2005, a partir de los datos seleccionados de los informes anuales de las empresas analizadas, la base de datos Hydra y el Banco Central de Malasia. Los resultados mostraron que la responsabilidad social empresarial tiene un impacto positivo en el desempeño financiero del mismo periodo. El cambio en el rendimiento financiero, en respuesta a los respectivos aumentos 0 reducciones de la responsabilidad social empresarial, es bastante evidente y significativo estadísticamente. Cuando el ROA y la $Q$ de Tobin se utilizan como variables dependientes en modelos de efectos fijos, la dimensión de producto tiene una relación positiva y significativa con el desempeño financiero. Sin embargo, la relación es negativa para la dimensión de medio ambiente y positiva en cuanto a ROA, pero negativa para la $Q$ de Tobin, en las dimensiones de relaciones con los empleados y compromiso con la comunidad. A la vista de los resultados de los modelos dinámicos, a largo plazo hay una limitada evidencia de una relación significativa entre responsabilidad social empresarial y desempeño financiero.

Durante 2004 y 2005, Makni, Francoeur y Bellavance (2009) estudiaron 179 empresas canadienses que cotizan en bolsa y usan las medidas de desempeño social empresarial, relacionadas la base de datos canadiense de inversión social (CSID, por sus siglas en inglés). Los resultados mostraron que las iniciativas sociales empresariales en Canadá, especialmente las relacionadas con los programas de medio ambiente, conducen a desempeños financieros pobres en el corto plazo. Comparadas con las grandes empresas estadounidenses, las empresas canadienses son relativamente pequeñas en tamaño. Las iniciativas medioambientales parecen demasiado costosas y no susceptibles de ser consideradas como inversiones sólidas por el mercado canadiense, y puede ser necesaria la ayuda gubernamental, a través de 
subsidios, para compensar el impacto negativo sobre el desempeño financiero que, a corto plazo, sufren las empresas.

Nelling y Webb (2009), en el periodo comprendido entre 1993 a 2000, examinaron la relación de causalidad entre la responsabilidad social corporativa y el desempeño financiero con una muestra 600 empresas estadounidenses tomando su información de la base de datos KLD. Usando el enfoque de datos de panel con efectos fijos, se encontró que la responsabilidad social empresarial global no es un predictor significativo del desempeño financiero. Visto en sentido contrario, es decir, en cuanto a los determinantes de la responsabilidad social global, parece existir un impacto positivo y significativo de la rentabilidad de los activos, aunque no de la rentabilidad bursátil. En cuanto a las calificaciones específicas de responsabilidad social según dimensiones, hay muy poca 0 ninguna evidencia de su impacto en el desempeño financiero. $Y$, por lo que atañe al análisis en dirección contraria, la rentabilidad bursátil conduce a una mayor calificación sólo en cuanto a relaciones laborales se refiere.

De otro lado, Ioannou y Serafeim (2010a) con una muestra de organizaciones de 42 países que pasó de 945 a 2.384 grandes empresas, desde el año 2002 hasta el año 2009, centraron la investigación en tres componentes: desempeño social, medioambiental y gobierno corporativo y concluyeron, en una visión global, que las características del país son determinantes más importantes del desempeño social empresarial que el sector de actividad de la firma, y este último es ligeramente más relevante que la propia compañía. Mostraron la existencia de una variación significativa en el desempeño social empresarial entre los diferentes países, toda vez que las firmas de unos países reciben altas puntuaciones en una dimensión de la responsabilidad social y las compañías de otros países las reciben en otra dimensión. Además, los principales determinantes del desempeño social y medioambiental en el nivel de país son las instituciones políticas, seguidas por las legales y del mercado de trabajo. En cambio, dentro de ese mismo nivel, en el gobierno corporativo son más las relevantes las instituciones legales que las políticas. Las instituciones del mercado de capitales parecen ser menos destacables.

Durante el año 2007, Fauzi e Idris (2010) estudiaron 220 empresas manufactureras de Indonesia que cotizaban en la bolsa de valores de Yakarta, con el propósito de determinar si el entorno empresarial, la estrategia de negocios, la formalización (establecimiento de comportamientos en el interior de la organización), la descentralización y otras variables relacionadas con la gestión moderan la relación entre la responsabilidad social empresarial y el desempeño financiero dentro las teorías del excedente de recursos y de la buena gestión. Los resultados mostraron que el entorno empresarial ha moderado el vínculo responsabilidad social empresarialdesempeño financiero en el contexto de la teoría de la buena gestión. La descentralización ha moderado la relación de la responsabilidad social empresarial y el desempeño financiero en el marco de la teoría del excedente de recursos. Así mismo, la interacción de algunas dimensiones del sistema de control ha moderado la relación de la responsabilidad social empresarial y el desempeño financiero en la teoría del excedente de recursos.

De manera similar, Choi, Kwak y Choe (2010) trabajaron con 1.222 empresas cotizantes en la Bolsa de Valores de Corea, en los años 2002-2008, con información proveniente del índice del Korea Economic Justice Institute (KEJI). Los resultados sugirieron que es importante para una empresa darse cuenta de qué aspecto de su responsabilidad social es más relevante para sus grupos de interés; y que las iniciativas sociales de una empresa, cuando son dirigidas apropiadamente, tienden a mejorar su cuenta de resultados.

A su vez, Humphrey, Lee y Shen (2010) investigaron los efectos de los factores medio ambiente, social y gobierno corporativo sobre el desempeño financiero en una muestra de las 249 empresas más grandes del Reino Unido que cotizan en bolsa. Existe muy poca evidencia de diferencias en el desempeño financiero en función de las carteras de acciones ordenadas según las puntuaciones en medio ambiente o gobierno corporativo. Sin embargo, se encontró una débil evidencia de que las empresas que invierten en iniciativas sociales tienen un desempeño inferior, aunque esto parece ser impulsado por el componente específico de las calificaciones sociales en los diferentes sectores. Tampoco parecen existir diferencias en cuanto a riesgo sistemático y ratio de valor contable/valor de mercado.

Así mismo, Brik, Rettab y Mellahi (2010) examinaron los efectos moderadores de la responsabilidad social empresarial sobre la asociación entre la orientación al mercado y el desempeño de la empresa en el contexto de una economía emergente en 310 empresas de diversos sectores, cuya información fue tomada de la base de datos de la Cámara de Comercio y la Industria de Dubái para el año 2009. En este estudio se concluyó que la responsabilidad social empresarial tiene un efecto sinérgico en relación con el impacto de la orientación al mercado sobre el desempeño del negocio.

Cavaco y Crifo (2010) se propusieron examinar los efectos de la responsabilidad social corporativa sobre el desempeño financiero de la empresa a partir de una muestra de 300 empresas que cotizan en bolsa, de 
diversos sectores y en 15 países europeos, durante el periodo 2002 - 2007. El impacto de las calificaciones de responsabilidad social empresarial sobre el rendimiento de la empresa depende del grado de complementariedad entre las diferentes tareas de responsabilidad social empresarial. Las calificaciones extra-financieras tienen un impacto positivo sobre los esfuerzos de responsabilidad social empresarial cuando por lo menos dos pares de tareas de responsabilidad social empresarial son complementarias. Cuando sólo un par de tareas de responsabilidad social son complementarias, entonces las calificaciones de responsabilidad social tienen un efecto ambiguo sobre el rendimiento de la firma.

Los investigadores loannou y Serafeim (2010b) estudiaron el impacto de las estrategias de responsabilidad social empresarial sobre las recomendaciones de los analistas de títulos. Se encontró evidencia de que, en los primeros periodos analizados, las estrategias de responsabilidad social empresarial fueron percibidas como destructoras de valor de las acciones y, por lo tanto, con un impacto negativo sobre las recomendaciones de los analistas de inversión. En cambio, para periodos posteriores, las fortalezas en responsabilidad social empresarial fueron percibidas como creadoras de valor, revirtiendo un impacto positivo sobre las recomendaciones. Las empresas con alta visibilidad tienen más probabilidades de recibir recomendaciones favorables cuando implementan estrategias de responsabilidad social empresarial, y estas últimas son mejor acogidas por los analistas con más experiencia y conocimientos de RSE.

Gallego y otros (2010) analizaron si crean valor las prácticas de responsabilidad social empresarial llevadas a cabo por las empresas europeas (tanto las prácticas de RSE relacionadas con estrategias de marketing como las que no lo son). La muestra de empresas se constituyó por las 120 mayores empresas europeas cuyas prácticas de RSE fueron analizadas por Deloitte and Kirchhoff Consult $(2007,2009)$. Los resultados obtenidos subrayan que todas las prácticas de RSE tienen un efecto positivo en la creación de valor para los accionistas, en especial aquellas de marketing relacionadas con la imagen de la compañía. Los inversionistas, a su vez, son capaces de detectar el nivel de compromiso de las empresas con el desarrollo sostenible. Por otro lado, ninguno de los tipos de prácticas de RSE analizados tiene una influencia significativa en la reputación corporativa. Este hallazgo puede sugerir cierto escepticismo con respecto a estos asuntos por parte de los diferentes actores y grupos de interés, tal vez debido a su incapacidad para diferenciar las prácticas reales de RSE.

El trabajo de Charlo y Moya (2010) se adelantó, con una muestra de 87 empresas españolas de diversos sectores durante el 2008, con el propósito central de comparar los principales parámetros financieros de las empresas socialmente responsables con sus equivalentes que no cumplen esta condición y analizar la posible existencia de diferencias significativas entre ambos grupos. Las empresas socialmente responsables obtienen una rentabilidad, adicional a su riesgo sistemático, superior a las empresas que no reúnen estos requisitos. Por lo tanto, es de esperar que esas empresas supongan una mejor oportunidad de inversión, al proporcionar más rentabilidad que la correspondiente a su riesgo.

Setó y Angla (2011) analizaron la relación entre la responsabilidad social empresarial y el desempeño financiero tanto desde la teoría de la buena gestión como desde la teoría del excedente de recursos, a partir de una muestra de 59 empresas españolas de diversas industrias. Los resultados sugieren la inexistencia de una relación significativa a corto plazo entre la responsabilidad social empresarial y el desempeño financiero, en ambas direcciones de la causalidad. Es decir, por un lado, las acciones de responsabilidad social empresarial adoptadas por la empresa no influyen en el resultado financiero a corto plazo, y por otro lado, el resultado financiero alcanzado por la empresa tampoco influye en la adopción de medidas de responsabilidad social empresarial. En consecuencia, no se confirma ni la teoría de la buena gestión ni la teoría del excedente de recursos.

A su vez, Rees (2011) a través de su investigación examinó el impacto de las participaciones estratégicas en el capital social y el financiamiento de la deuda sobre el desempeño de la empresa en aspectos medioambientales, sociales y de gobierno corporativo. En la muestra se fue incrementando el número de empresas año a año, comenzando con 961 en el 2002 hasta llegar a 3.353 de 56 países en el año 2009. El efecto de los inversores institucionales tales como los fondos de pensiones es poco claro, en cambio, tiene un impacto positivo la participación accionarial del gobierno del país, junto con la deuda. Los accionistas "atrincherados", ya sean empleados y familiares o participaciones corporativas cruzadas, se asocian negativamente con las calificaciones en materia de responsabilidad social corporativa.

Andersen y Dejoy (2011) investigaron el papel de las frecuentemente especificadas variables de control en la moderación de la relación entre el desempeño social de las empresas y su rendimiento financiero, en una muestra de 550 empresas de Estados Unidos. Los resultados de este estudio apoyan la conclusión de una relación positiva a largo plazo entre desempeño social empresarial y desempeño financiero. Además, también se demuestra la necesidad de incluir variables de control adecuadas. Las ventas y el sector se reconocen como importantes variables que afectan a la relación entre el desempeño social empresarial y su desempeño financiero. 
Cheng, loannou y Serafeim (2011) investigaron si un comportamiento superior en las estrategias de responsabilidad social empresarial, conduce a un mejor acceso a la financiación. Las empresas que hicieron parte de la muestra pertenecen a 49 países a través del mundo, las cuales fueron incrementándose durante los años observados, pasando de 486 a 2.191 durante los años 2002 a 2009. Se comprueba la hipótesis de que un mejor acceso a la financiación es atribuible a la reducción de los costos de agencia, debido a una mayor participación de los grupos de interés a través de la responsabilidad social empresarial, y la reducción de las asimetrías de información, debido a una mayor transparencia a través de informes no financieros. Las empresas con mejor responsabilidad social empresarial son las que tienen menores restricciones de capital. La responsabilidad social empresarial tiene un impacto significativo sobre el proceso de asignación de capital descentralizado: los participantes en el mercado están más dispuestos a asignar recursos de capital a empresas con mejor desempeño en responsabilidad social empresarial.

El estudio realizado por Marsat y Williams (2011) analizó específicamente la relación entre el valor de mercado de la compañía y la responsabilidad social empresarial, cualesquiera que sean las razones subyacentes de los inversores, a partir de una muestra de 2.838 empresas de diferentes países. La mayor parte del trabajo empírico académico informa de una relación positiva entre el desempeño en la responsabilidad social empresarial y el valor de la empresa, sin embargo, este estudio encuentra un efecto negativo. El resultado parece ser robusto, ya que no se ve afectado por: (1) un cambio en la variable dependiente que expresa el valor de la empresa, (2) la incorporación de variables adicionales (investigación y desarrollo e investigación y desarrollo multiplicada por el crecimiento de las ventas) y (3) un cambio en la representación de la responsabilidad social empresarial.

Barnett y Salomon (2012) pretendieron demostrar, a través de un estudio efectuado entre 1998 y 2006, que la relación entre el desempeño social empresarial y el desempeño financiero no es linealmente positiva 0 negativa, sino que existe una relación curvilínea. La muestra fue compuesta por un panel de datos no balanceado de 1.214 empresas estadounidenses provenientes de diversos sectores. Se encontró una relación en forma de $U$ entre el desempeño social y el desempeño financiero de la empresa, también se descubrió que las empresas con más alto desempeño social empresarial generalmente tienen más alto desempeño financiero. Algunas empresas son socialmente más responsables que otras porque enfrentan condiciones del mercado que demandan más responsabilidad social.
En cambio, si los directivos son conscientes de que su firma tiene menos capacidad que sus competidores para influir sobre sus grupos de interés a través de la actuación social, entonces pueden optar por la racional decisión estratégica de ser menos responsables socialmente.

Carnevale, Mazzuca y Venturini (2012) buscaron proponer una clave para entender la relación entre la información social y el valor que el mercado atribuye a los bancos que dan a conocer su compromiso con la responsabilidad social empresarial a través de informes sociales. La muestra correspondió a 130 bancos europeos cotizados en bolsa pertenecientes a la zona Euro-12, realizando el análisis para los años 2002 a 2008. Los estadísticos descriptivos muestran que tanto el precio de las acciones como sus valores contables son más altos para los bancos que publican un informe social. Los reportes sociales son más comunes entre los bancos que generan más beneficios y tienen más recursos propios. El precio de las acciones de los bancos que publican un reporte social parece mostrar una menor variabilidad.

A su vez, Martínez-Ferrero y Frías-Aceituno (2013) buscaron aclarar la relación entre el comportamiento sostenible de las empresas y su desempeño financiero, con una muestra de 1.960 empresas multinacionales no financieras cotizadas en bolsa y pertenecientes a 25 países. La información de responsabilidad social empresarial fue tomada de la base de datos EIRIS (Empowering Responsible Investment). Los resultados indican que las empresas con un nivel superior de desempeño financiero asignan más recursos a la mejora de las prácticas sostenibles los cuales se centran en los aspectos sociales y la mejora de sus relaciones con las partes interesadas; esto se traduce en un mejor rendimiento general debido a los impactos de la responsabilidad social empresarial en la reputación de la empresa, que generan un mejor desempeño de la empresa.

\section{CONCLUSIONES:}

Las primeras investigaciones analizadas giraron alrededor de aspectos de responsabilidad social empresarial 0 desempeño social empresarial (conceptos que se han manejado de modo indiferente) y la relación con el desempeño financiero o valor de mercado de las empresas a partir del estudio de las acciones sociales y medioambientales de las organizaciones, como variables independientes, y en algunos casos se consideraron, igualmente, aspectos relacionados con el gobierno corporativo. Las variables dependientes, por su parte, se referían a los resultados financieros de las compañías. 
Por otro lado, las investigaciones más recientes sobre la temática de estudio, se están enfocando hacia el impacto de todas las vertientes de la ESG1 en el desempeño financiero de las empresas. Sin embargo, en los resultados finales 0 conclusiones de los estudios reseñados se evidencia, en términos generales, una alta presencia de los que indican que existe una relación positiva entre la responsabilidad social empresarial y el desempeño financiero, unos pocos manifiestan que esa relación no es fuerte o relevante, $y$ tan solo en uno de ellos señala que es negativa.

Es importante mencionar, igualmente, el alto grado de coincidencia en cuanto a las variables utilizadas para la realización de los estudios empíricos; en lo que respecta a los indicadores de responsabilidad social empresarial se destacan los ratings que ofrece la base de datos de KLD, aunque algunos estudios no trabajaron con los ocho componentes de dicha base, manejando solo los más comunes: relación con los empleados, producto, relaciones con la comunidad, medio ambiente, y tratamiento a las mujeres y minorías. En cuanto a la técnica estadística 0 econométrica utilizada, se observa en gran medida el uso de los mínimos cuadrados ordinarios y el método generalizado de los momentos (GMM, por sus siglas en inglés). En muchos de los estudios simplemente se menciona como técnica de estimación, la utilización de la regresión lineal.

Finalmente, en las limitaciones que presentaron algunas de las investigaciones, se aprecia el sesgo en el momento de seleccionar la muestra, ya que dependen de la información depositada en las bases de datos, lo cual no permite hacer una selección por muestreo aleatorio de las empresas que participarán en el estudio. En aquellos casos en los que se decidió obtener la información directamente de las organizaciones, los investigadores se encontraron con inconvenientes en cuanto a la respuesta oportuna de los instrumentos de recolección de datos y la tabulación de los mismos.

\section{REFERENCIAS:}

Aguilera, J., Guerrero, J. y Morales, M. (2015). Responsabilidad social y desempeño financiero en multinacionales: influencia de la diversificación internacional. Revista Venezolana de Gerencia, año 20, No. 71, pp. 419 - 439.

\footnotetext{
${ }^{1}$ Siglas en inglés que corresponde a Environmental, Social y Governance; es decir, se integran los aspectos medio ambientes, sociales y de gobierno corporativo.
}

Andersen, M. L. y Dejoy, J. S. (2011). Corporate social and financial performance: the role of size, industry, risk, R\&D and advertising expenses as control variables. Business and Society Review, vol. 116, pp. 237 - 256.

Aupperle, K., Carroll, A. y Hatfield, J. (1985). An empirical examination of the relationship between corporate social responsibility and profitability. Academy of Management Journal, vol. 28, pp. 446 - 463.

Barnett, M. y Salomon, R. (2006). Beyond dichotomy: the curvilinear relationship between social responsibility and financial performance. Strategic Management Journal, vol. 27, pp. 1101 - 1122.

Barnett, M. y Salomon, R. (2012). Does it pay to be really good? Addressing the shape of the relationship between social and financial performance. Strategic Management Journal, vol. 33, pp. 1304 $-1320$.

Bouquet, C. y Deutsch, Y. (2008). The impact of corporate social performance on a firm's multinationality. Journal of Business Ethics, vol. 80, pp. 755 769.

Brik, A. B., Rettab, B. y Mellahi, K. (2010). Market orientation, corporate social responsibility, and business performance. Journal of Business Ethics, vol.99, pp. 307-324.

Carnevale, C., Mazzuca, M. y Venturini, S. (2012). Corporate social reporting in European banks: the effects on a firm's market value. Corporate Social Responsibility and Environmental Management, vol. 19, pp. $159-177$.

Cavaco, S. y Crifo, P. (2010). The CSR-firm performance missing link: complementarity between environmental, social and business behavior criteria? Consulta 1-6-2012, en http://hal.archives-ouvertes.fr/hal-00504747/

Charlo Molina, M. y Moya Clemente, I. (2010). El comportamiento financiero de las empresas socialmente responsables. Investigaciones Europeas de Dirección de la Empresa, vol. 16, pp. $15-25$.

Cheng, B., loannou, I. y Serafeim, G. (2011). Corporate social responsability and acces to finance. Harvard Business School Research Paper No. 1847085. 
Choi, J., Kwak, Y. y Choe, C. (2010). Corporate social responsibility and corporate financial performance: evidence from korea. Australian Journal of Management, vol. 35, pp. 291 - 311.

Cochran, P. y Wood, R. (1984). Corporate social responsibility and financial performance. Academy of Management Journal, vol. 27, pp. 42 -56 .

D’Arcimoles, C. y Trébucq, S. (2003). Etude de l'interaction entre performance financière et performance sociétale: le cas des sociétés françaises cotées (1998-2000). Working paper No. 2003-106, disponible en http://cermat.iae.univtours.fr/IMG/pdf/CAHIER 2003106_D ARCIMOL E S.pdf.

Fauzi, H. e Idris, K. M. (2010). The effect of contextual variables in the relationship between CSR and CFP: evidence from indonesian companies. Social and Environmental Accounting, vol. 4, pp. $1-25$.

Gallego, I., Prado, J., Rodríguez, L. y García, I. (2010) Are social and environmental practices a marketing tool? Management Decision, Vol. 48 pp. 14401455.

Gitman, L., J. y Zutter, Ch., J. (2012). Principios de administración financiera. Pearson Educación. México

Humphrey, J., Lee, D. y Shen, Y. (2010). The independent effects of environmental, social and governance initiatives on the performance of UK firms. 23rd Australasian Finance and Banking Conference. Sydney, 15 - 17 de diciembre

Ioannou, I. y Serafeim, G. (2010a). What drives corporate social performance? International evidence from social, environmental and governance scores. Harvard Business School, Working paper No. 11016.

loannou, I., y Serafeim, G. (2010b). The impact of corporate social responsibility on investment recommendations. Harvard Business School, Working paper No. 1507874.

Makni, R., Francoeur, C. y Bellavance, F. (2009). Causality between corporate social performance and financial performance: evidence from Canadian firms. Journal of Business Ethics, vol. 89, pp. 409 -422 .
Marsat, S. y Williams, B. (2011). CSR and market valuation: international evidence. Conference of the French Finance Association (AFFI), Paris, 11 -13 de mayo.

Martínez-Ferrero, J y Frias-Aceituno, J. (2013) Relationship between sustainable development and financial performance: international empirical research. Business Strategy and the Environment. doi: 10.1002/bse.1803

Mcguire, J., Sundgren, A. y Schneeweis, T. (1988). Corporate social responsiblity and firm financial performance. Academy of Management Journa, vol. 31, pp. 854 - 872.

Nelling, E. y Webb, E. (2009). Corporate social responsibility and financial performance: the "virtuous circle" revisited. Review of Quantitative Finance and Accounting, vol. 32, pp. 197 - 209.

Ortiz, H. (2015). Análisis financiero aplicado. Universidad Externado de Colombia. Bogotá

Preston, L. E. y O'Bannon, D. P. (1997). The corporate social-financial performance relationship. Business and Society, vol. 36, pp. 419 - 429.

Rappaport, A. (2006). La creación de valor para el accionista. Ediciones Deusto. Barcelona

Rees, W. (2011). Investor Influence on firms' environmental, social and governance performance. Working paper No. 1973724, disponible en http://ssrn.com/abstract=1973724.

Ruf, B. M., Muralidhar, K., Brown, R. M., Janney, J. J. y Paul, K. (2001). An empirical investigation of the relationship between change in corporate social performance and financial performance: a stakeholder theory perspective. Journal of Business Ethics, vol. 32, pp. 143 - 156.

Saleh, M., Zulkifli, N. y Muhamad, R. (2008). An empirical examination of the relationship between corporate social responsibility disclosure and financial performance in an emerging market. Disponible en http://www.pbfeam2008.bus.qut.edu.au/papers/d ocuments/MustaruddinSaleh_Final.pdf

Setó Pamies, D. y Angla Jimenéz, J. (2011). La naturaleza de la relación entre la responsabilidad social empresarial (RSE) y el resultado financiero. Revista Europea de Dirección y Economía de la Empresa, vol. 20, pp. 161 - 176. 
Waddock, S. y Graves, S. (1997). The corporate social performance- financial performance link.

Strategic Management Journal, vol. 18, pp. 303 319.

Ziegler, A., Rennings, K. y Schröder, M. (2002). The effect of environmental and social performance on the shareholder value of european stock corporations. Centre for European Economic, Working paper, disponible en http://www.eea-

esem.com/papers/eeaesem/2003/342/ paper.pdf 